\title{
Road Traffic Monitoring System
}

\author{
G. Shobana, M. Suguna
}

\begin{abstract}
Nowadays, the Congestion in traffic is one of the major issues in many cities. There are different causes of congestion in road traffic like capacity of the lane is insufficient, more demand, large delays in signals, increase the number of vehicles etc. As populations are growing, it is difficult to manage the traffic on the roads and also the people has to wait for the green signal for a long time as it is not depend on the density of traffic in the real time. This paper presents the method to find the density of the traffics in each signal by using camera and sensors. According to the vehicle density on road, the decisions are made to switch the traffic lights to red or green. As the result it will reduce the traffic on roads which will help to reduce the accidents
\end{abstract}

Keywords: Density of traffic, Internet of Things, Congestion, Sensors

\section{INTRODUCTION}

As increase in the population, the usage of road is increased day by day. The managing of the traffic is one of the major challenges to be tackled in a big city. The usage of vehicles rise to congestion of the road traffic which will leads to increasing cost of transportation because of wastage of time and extra fuel consumption due to waiting in the traffic signal. The most common reason of traffic congestion in the world is an inefficient traffic signal controlling which affects the traffic flow. So the traffic signal has to be changed based on the current traffic in the lane. In the existing traffic system in our cities, predefined timers are used in the traffic signal for controlling the traffic. It will not consider for real time scenario of traffic in the roads. As result the green signal is for lane with no vehicle and lot of vehicle has to be wait in the red signals because same wait timing is used for all the lanes.

India loses billions of money in the year 2016, due to lot of traffics which will leads to wastage of fuel, vehicles speed is reduced and waiting time of vehicles at toll plazas are increased. The most road traffic congestion city are Mumbai, Pune, Kolkata are statics of the traffic given by "The Times of India". The system has to developing order to handle the traffic in the India. The Internet of Things plays important roles in sensing the road traffics and make intelligent decision on that current situation. IOT is connecting with the devices by sense the data, send the information to other end, after that it process based on that decision are made.

Analysis of the transportation data are helpful for project planning for developing the Intelligent system in transport by using the vehicle count, speed, types of vehicles etc. For estimating the traffics in the road, sensors are used for

Revised Manuscript Received on 14 August, 2019.

G.Shobana, Assistant Professor, Department of Computer Science and Engineering,Kumaraguru College of Technology, Coimbatore, Tamilnadu, India.

Dr.M.Suguna, Assistant Professor, Department of Computer Science and Engineering, Kumaraguru College of Technology, Coimbatore, Tamilnadu, India. monitoring traffic and Information system related to classification of vehicles. In the current situation, vehicles count can be obtained by magnetic loop detectors. The count is calculated by passing the vehicle over them. Other parameter for traffic monitoring such as vehicle classification, size are obtained by Vision-based video monitoring systems. In the metropolitan city, the monitoring the traffic can be done by video camera which is mounted in the poles which will focus on the each lane and record the traffic in each lane. It will be used for detecting and classifying vehicles in different lanes and for any direction of traffic flow. This information will be helpful for analysis of the traffic in particular areas.

In today's world, the signals are working in each road. In the system, vehicles in one path (P1) are passed and vehicles in another path (P2) still in waiting state because time is not over and hence signal is still red. These systems are not efficient to handle the present situation. The main drawback in the existing system has unnecessary waiting time even though there is less number of traffic in the road and there is no facility to handle emergency vehicles in the traffic. The system is developed to handle the situation, which performs execution based on density of vehicles in the each path. Based on number of vehicle the signals will be allotted for a particular side. For processing this scenario sensors and Arduino is used as a microcontroller which provides the signal timing based on the traffic density.

The paper is organized as Section II describes literature survey on the traffic management system Section III describe our proposed system Section IV describes the simulation model with hardware setup and finally Section V concludes the paper with a highlight on the scope of future work

\section{II.LITERATURE SURVEY}

Internet of things plays important roles in today's world. It has gained significant attention in computing industry during these years. It is used to control and manage the traffic system more effectively. But each systems use different types of technologies, different types of sensors and other different components like Infrared Sensors, Radiofrequency identification tags, camera, Big Data, Zigbee, Bluetooth etc.

Now a day's usage of vehicle is more when compared to the past decades. Infrastructure growth of the road is less as compared to the increase in number of vehicles, due to some constraints like space and cost [3]. Traffic system that we follow is fixed time is allotted to each path. Even though there will be lot of vehicle has to be wait for more one signal at particular area to pass vehicle from that particular signal.[1]

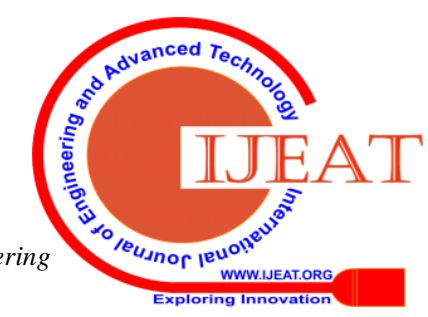


HarshiniVijeth (2017) had suggested method which uses Infrared sensors and RFID are used to identify the vehicle count on each side of signal and also to identify the ambulance. Camera which is mounted on poles is used to capture the picture of traffic. Decision can be made manual after seeing the picture of traffic in control room. Demerits of this system, camera will cover minimal area of signals. Pi cameras are used in this system [7].

Bilal Ghazalhas (2016) proposed method, the density of the vehicle using IR sensors and accomplishes dynamic timing slots with different levels. Moreover, a portable controller device is designed to solve the problem of emergency vehicles stuck in the overcrowded roads. In many traffic monitoring system, IR sensors are used [8]. The direction of vehicle is identified by IR transmitter and the IR receiver that are mounted on poles on either sides of the road .When an vehicles passes on the path between the IR sensors, it will increase the vehicle count. The based on information collected about the traffic density of the different roads of a junction is analyzed and decision can be made. PIC microcontroller is used to control the traffic system. Sensing of vehicles by IR rays has disadvantage that it can't able detect automobiles properly due to small blocks such as ranging from rain droplets to fog and dust particles etc.

KoushikMandal (2012) had proposed the system in RFID and GSM technology are used to get real time information from the traffic and signals are changed according it. RFID tags are fixed on the each automobile. It has Wireless router and wireless coordinator which will read RFID that is placed in vehicle. RFID reader is placed in the pole on either side of road. Based on the information obtained, average waiting time is calculated and GSM module helps to transmit the data to the central traffic management system for making decision.[9]

Siuli Roy (2016) had proposed the system in which the speed of the vehicles are identified.[8] speed of the vehicle is obtained by RFID and other sensors. If the average speed of vehicles falls below a threshold which is calculated earlier based on the traffic information at the particular area, automatically the system will detect that traffic congestion are occurred by comparing the with the normal traffic with speed of vehicle. In such a situation, a message is sent to the central area for asking to stop all inflow of traffic in that signals. This system has some manual operation for managing the traffic

\section{III.PROPOSED SYSTEM}

The proposed system helps to reduce the possibilities of traffic congestion in the roads, which is caused by the traffic lights signals, to some extent by clearing the road with contains high density of vehicles. It also provides the clearance for the emergency vehicle by capturing the images from path. The process of the maintaining the traffic in the roads is shown in the figure 3.1

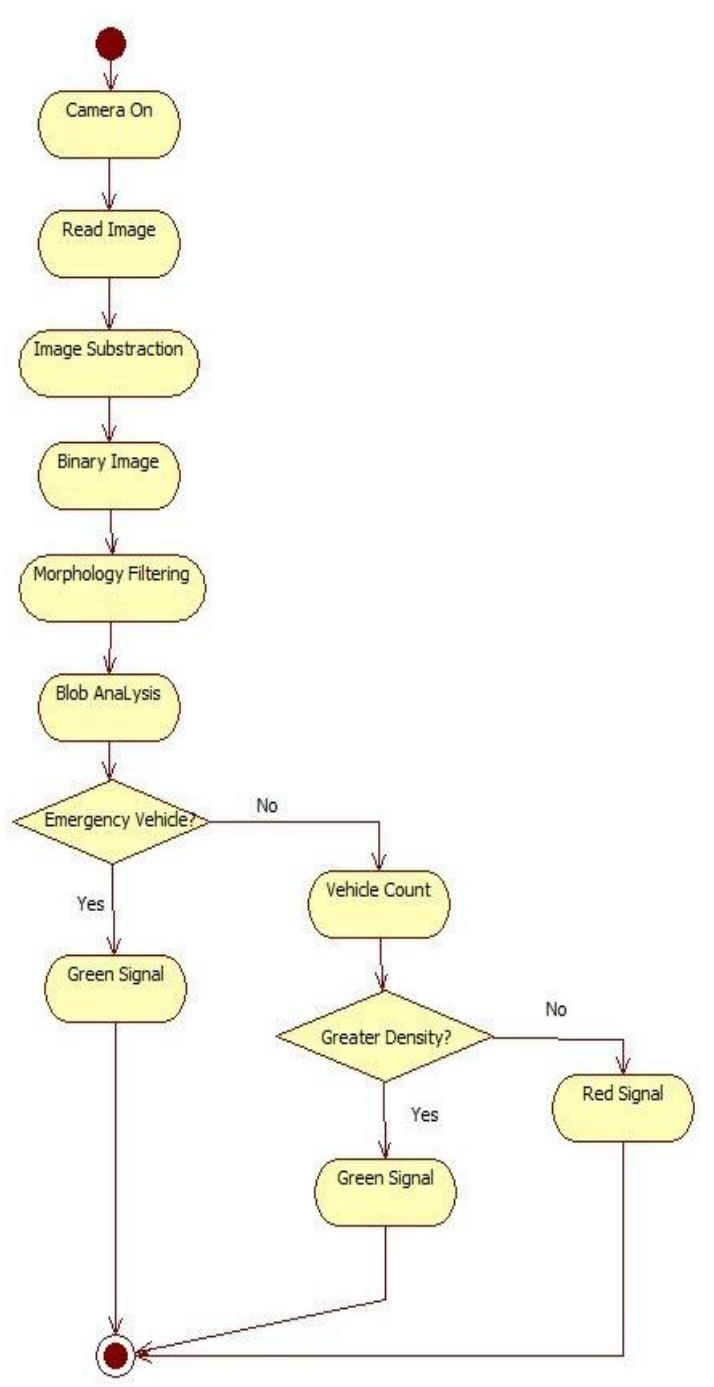

Figure 3.1: Architecture of the proposed system

\section{Image acquisition}

The images of each lane are captured by the video camera which is placed on the top of the pole which is near the traffic signal. From which it can able to capture the image of vehicle clearly. The information are extracted from video camera, from that images are separated into the frames continuously from the real time. The data are processed by converting the images from RGB format to grayscale for processing. Reference image are image which are captured during no vehicle present in the road.

\section{Image Pre-Processing}

The continuously record the traffic video from the each Lanes. Consider one frame per second from video using image processing. The unnecessary background information from the video images is removed. In the system initial background image without vehicles are stored that is subtracts from the current image of traffic. The dimensions of the road are already calculated. It performs Image Filtering. It uses two processes such as roads are converted to white and other that is converting to black. 


\section{Finding Traffic Density}

Vehicle detection is required to identify type of vehicles and count of vehicle, which are present in particular area. Identification of vehicles can be done by rectangular area. If more than one area is collapsed then it will be filter out. After finding the number of vehicle in the each lane, the signal can be changed by comparing with the other lanes vehicle density. If it find any emergency vehicle in the lane then signal can be changed according to it.

\section{RESULTS}

In the system four corner signals are taken for analysis of traffic system. The hardware setup of arduino with video camera are used for capturing traffic video image are shown in the figure 4.1 The vehicle counts are identified in each lane by capturing video. Vehicle count when compared with other lane. Based on analysis the green signals are opened to that particular lane. In this figure 4.2 signals 2 has 4 vehicles and signal 4 has 6 vehicles. After comparing the values, the green signal is displayed to signal 4.

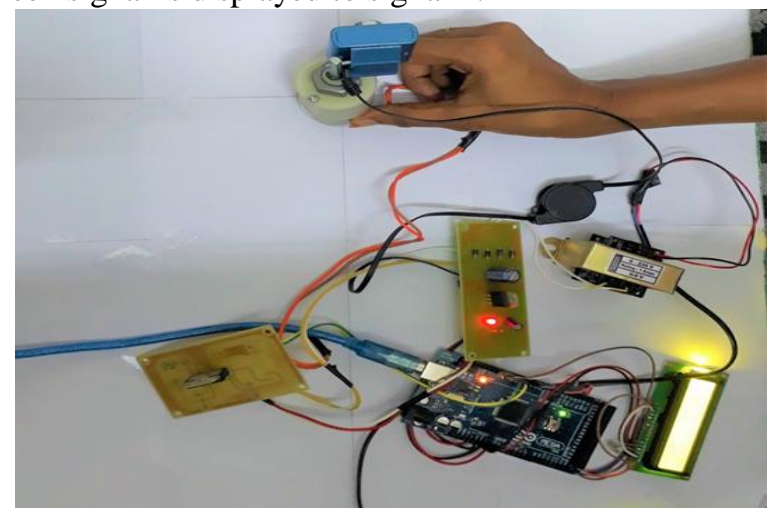

Figure 4.1: Hardware Setup

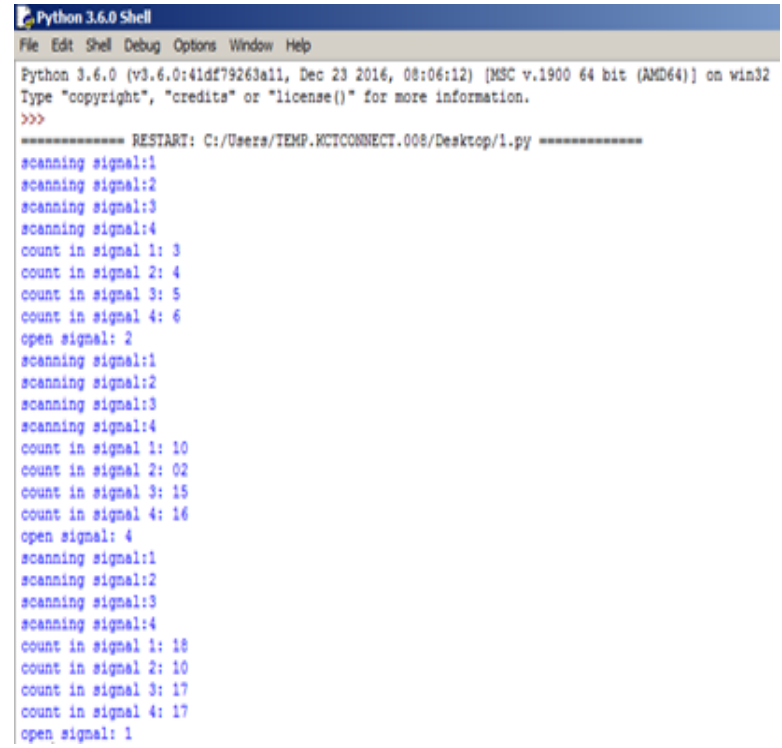

Figure 4.2 Simulation Results of Signal

\section{V.CONCLUSION}

The system reduces the possibilities of traffic congestion in the roads. By monitoring the vehicle density in each path in the road and based on real time traffic congestion green signal are changed accordingly. Information obtained from traffic signals are stored for analysis of the traffic particular area for project planning. Due to the climate changes video capturing of images vehicle may not able clear. So in the future this can be identified.

\section{REFERENCE}

1. S.Lokesh, and T.Prahlad Reddy, 'An Adaptive Traffic Control System Using Raspberry PI' National Conference On Nextgen Technology(ICNET),978-15090-6342-7/17/\$31.00@IEEE, 2015

2. R.NithinGoutham, J. Sharon Roza and M.Santhosh,'Intelligent Traffic Signal Control System'International Conference On Invotaions in Engineering and Technology(ICIET'14) , 948-1-42368563-4/14@2014

3. Ms.PallaviChoudekar, Ms.Sayanti Banerjee , and Prof.M.K.Muju, 'Real Time Traffic Light Control Using Image Processing' National Conference On Internet Of Things(IOT),978-1-4682-9586-3/13,2013

4. Priyanka Khankeand, Prof. P. S. Kulkarni, 'A Technique on Road Tranc Analysis using Image Processing' IEEE National Conference on Product Design (NCPD'14), 978-1-4673-9448-7/17,2014

5. Rajeshwari Sundar, SanthoshsHebbar, and VaraprasadGolla(2015) ， 'Implementing intelligent Traffic Control System for Congestion Control' International Journal on Inovations in Engineering and Technology,738-1-4673-9445-2/18@2015

6. Vidhya and Bazile Banu 'Density Based Traffic Signal System' IEEE International Conference On Inovations in Engineering and Technology (ICIET'14), 978-1-46739448-7/17, 2014.

7. Harshini Vijeth ,Dr. Nataraj K R,'IOT Based Intelligent Traffic Control System", International Journal For Research In Applied Science \&Engineering Technology,2017

8. Bilal Ghazal, Khaled ElKhatib , "Smart traffic light control system" in IEEE conference with ISBN: 978-14673-6941-1 , 2016

9. Koushik Manda, "Road Traffic Congestion Monitoring and Measurement using Active RFID and GSM Technology” 2012 